Abstract 411 Table 1 The prevalence of SLE in South Korea (per 100,000).

\begin{tabular}{llllll}
\hline & 2010 & 2011 & 2012 & 2013 & 2014 \\
\hline Total & 33.9 & 36.1 & 37.7 & 41.5 & 44.6 \\
Male & 8.4 & 9.2 & 9.6 & 11.5 & 13.1 \\
Femal & 59.6 & 63.3 & 65.9 & 71.7 & 76.3 \\
\hline
\end{tabular}

411 THE PREVALENCE OF SYSTEMIC LUPUS ERYTHEMATOSUS HAS INCREASED IN SOUTH KOREA

K Moon*. Kangwon national university hospital, Internal Medicine, Chuncheon-SiGangwon-Do, Republic of Korea

\subsection{6/lupus-2017-000215.411}

Background and aims Systemic Lupus Erythematosus (SLE) is an autoimmune disease with diverse clinical manifestations. Despite of progression of understanding and treatment of SLE, there is scarcity of data about SLE in Asia. We evaluated the prevalence of SLE for 5 years from 2010 to 2014 in South Korea using data from the Health Insurance Review and Assessment Service (HIRAS). The purpose of this study is to investigate the prevalence of SLE in South Korea.

Methods We used data provided by HIRAS, which covers all hospital visits nationwide. Identification of patients with SLE was based by main diagnostic code. The prevalence of SLE was calculated for 5 years from 2010 to 2014 .

Results The prevalence of SLE has increased for 5 years. The prevalence rate of SLE was 33.9/100,000 (male 8.4/100,000, and female $59.6 / 100,000)$ in 2010 , but it has increased to 44.6/100,000 (male 13.1/100,000, and female 76.3/100,000) in 2014.

Conclusions The prevalence of SLE has increased from 2010 to 2014 in South Korea.

\section{2 MORTALITY IN CHINESE PATIENTS WITH SYSTEMIC LUPUS ERYTHEMATOSUS}

L Mu, Y Hao*, H Huang, A Xie, X Zhang, L Ji, Y Geng, X Deng, J Zhao, G Li, Y Wang, W Zhou, Z Zhang. Peking University First Hospital, Department of Rheumatology and Clinical Immunology, Beijing, China

10.1136/lupus-2017-000215.412

Background and aims To investigate the mortality and the causes of death in Chinese patients with systemic lupus erythematosus (SLE).

Methods We collected the clinical data of all 950 SLE patients in Rheumatology department of Peking University First Hospital from May 2007 to January 2016.The primary causes of death were identified, the standardised mortality ratio (SMR) and years of life lost (YLL) were calculated based on the National Bureau of Statistics of China for the general population, and the survival was determined by Kaplan-Meier analysis.

Results The mean age of all 950 patients (849 females and 101 males) was $37.9 \pm 14.8$ years old, the median disease duration at recruitment was $2.7(0.5-7.0)$ years, and the median follow-up duration was $4.7(2.2-8.4)$ years. Among 881 patients who were successfully traced, 45 patients died. Infection (31.1\%) was the leading cause of death followed by lupus nephritis, pulmonary arterial hypertension (PAH) and cerebrovascular disease (Table 1). The overall age and sex adjusted SMR was 3.3 (95\%CI 2.3-4.3), and the age adjusted SMR of women and men were $5.1(3.6-6.7)$ and $1.3(0.0-$ 2.6) respectively. The YLL for women and men were 18.1 and 15.0 years respectively (Table 2). The overall survival at 1,5 and 10 years were $98.2 \%, 95.3 \%$ and $93.7 \%$ respectively. The survival of patients with $\mathrm{PAH}$, thrombocytopenia and hemolytic anaemia were significantly worse than those without these complications (Figures 1, 2 and 3).

Conclusions Mortality of SLE patients in China is substantial, especially in female, and infection is the leading death cause. Patients with $\mathrm{PAH}$, thrombocytopenia and hemolytic anaemia had worse prognosis.

Abstract 412 Table 1 Causes of death.

\begin{tabular}{llll}
\hline $\begin{array}{l}\text { SLE related cause of death } \\
\mathrm{n}=13\end{array}$ & $\mathrm{~N}(\%$ in 45$)$ & $\begin{array}{l}\text { Non-SLE related Cause of death } \\
\mathrm{n}=23\end{array}$ & $\mathrm{~N}(\%$ in 45) \\
\hline Lupus nephritis & $3(6.7)$ & Infection & $14(31.1)$ \\
Pulmonary arterial hypertension & $3(6.7)$ & Cerebrovascular disease & $3(6.7)$ \\
NeuropsychiatricSLE & $2(4.4)$ & Ischemic heart disease & $1(2.2)$ \\
Thrombocytopenia & $1(2.2)$ & Liver failure & $1(2.2)$ \\
Hemolytic anemia & $1(2.2)$ & Gastrointestinal bleeding & $1(2.2)$ \\
Alveolar hemorrhage & $1(2.2)$ & Acute pancreatitis & $1(2.2)$ \\
Myocardial Involvement & $1(2.2)$ & Malignancy & $1(2.2)$ \\
Gut and urinary smooth muscle & $1(2.2)$ & Suicide & $1(2.2)$ \\
Involvement & & & \\
\hline
\end{tabular}

Note: In total 45 died patients, the cause of death could not be determined in 9 (20\%) patients. 
Abstract 412 Table 2 The standardized mortality ratio (SMR), life expectancy (LE) and years of life lost (YLL).

\begin{tabular}{llll}
\hline & $\begin{array}{l}\text { Overall } \\
\mathrm{n}=950\end{array}$ & $\begin{array}{l}\text { Female } \\
\mathrm{n}=849\end{array}$ & $\begin{array}{l}\text { Male } \\
\mathrm{n}=101\end{array}$ \\
\hline Death number & 45 & 41 & 4 \\
SMR $(95 \% \mathrm{CI})$ & $3.3(2.3-4.3)$ & $5.1(3.6-6.7)$ & $1.3(0.0-2.6)$ \\
LE of general population (years) & - & 83.5 & 79.8 \\
LE of SLE patients(years) & - & 65.4 & 64.8 \\
YLL (years) & - & 18.1 & 15.0 \\
\hline
\end{tabular}

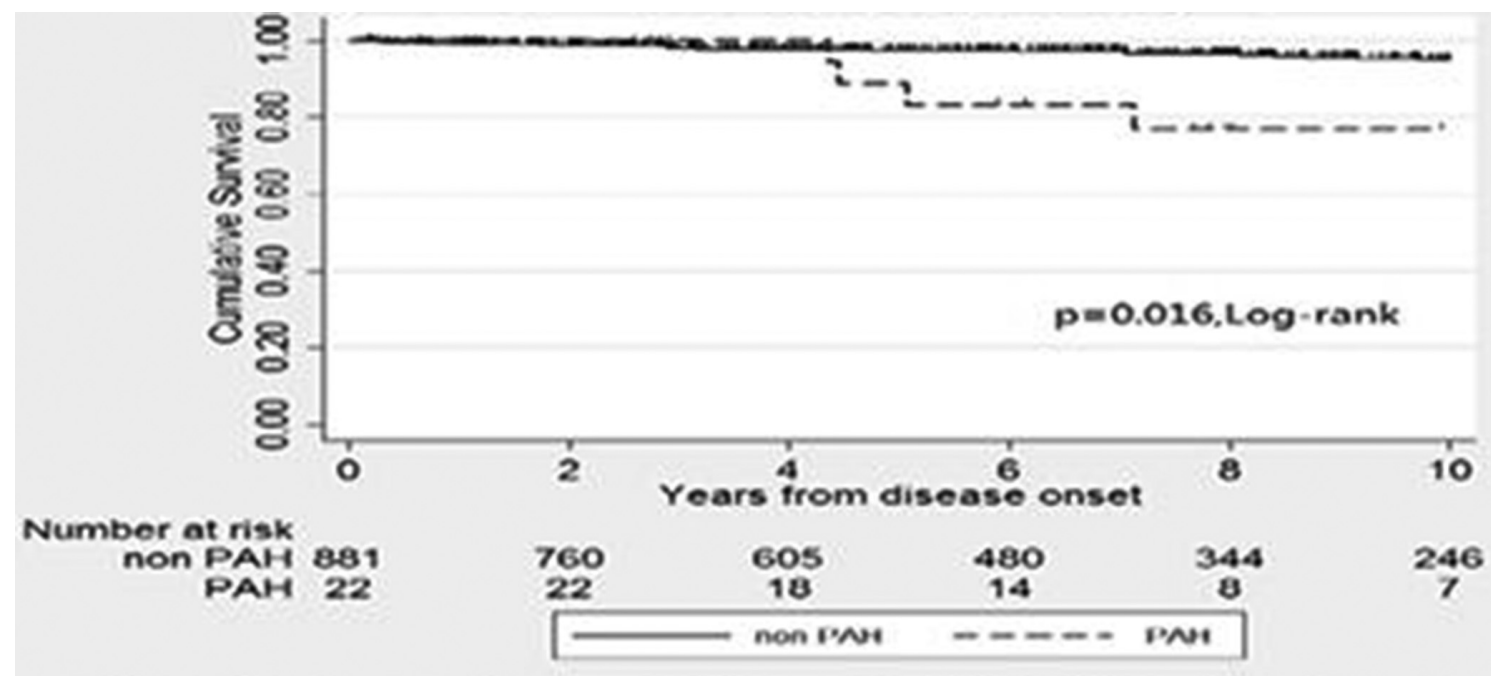

Abstract 412 Figure 1 Survival curve of SLE patients by PAH.

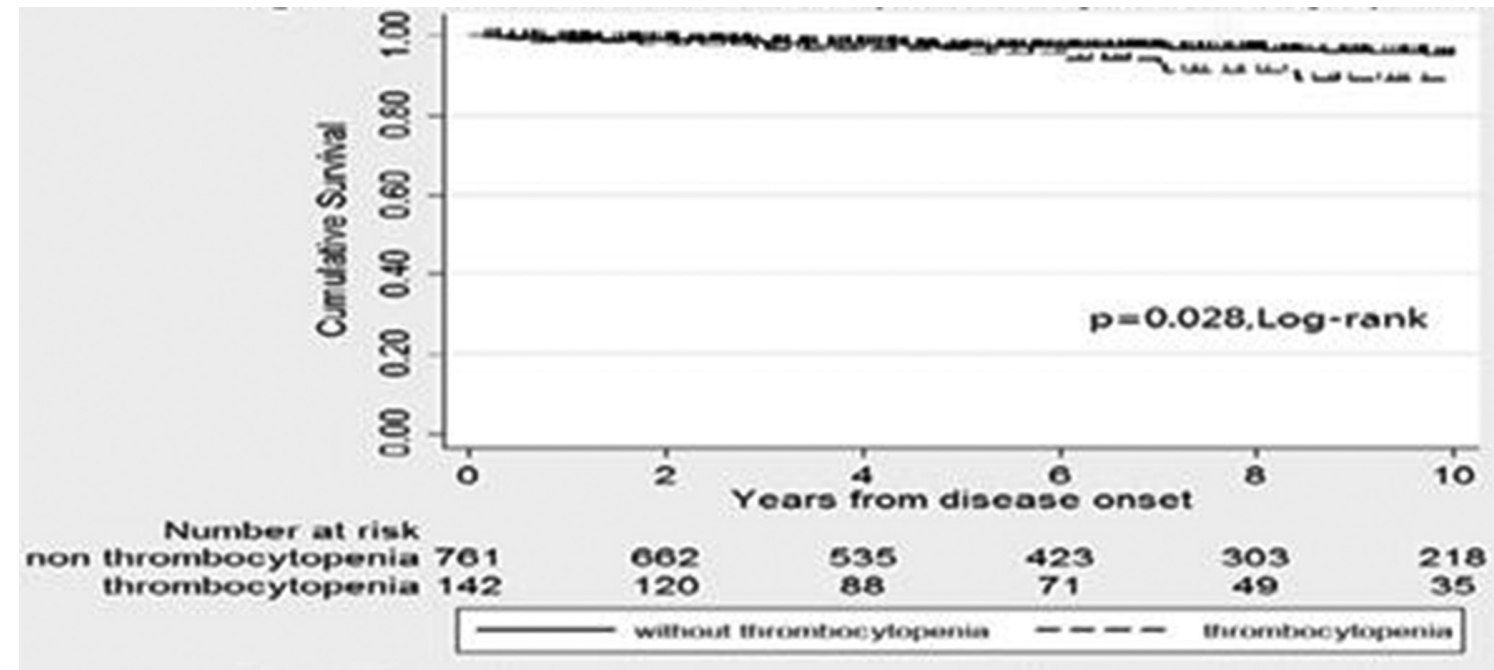

Abstract 412 Figure 2 Survival curve of SLE patients by PAH. 


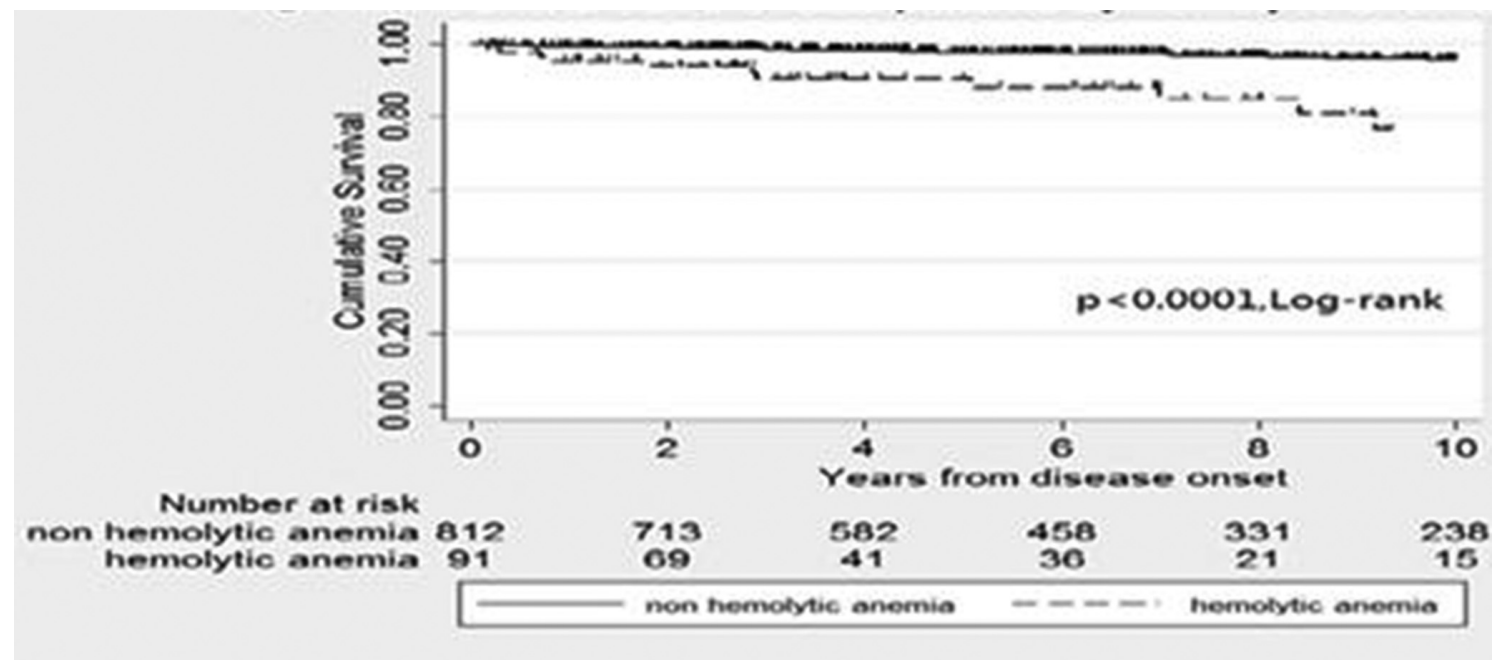

Abstract 412 Figure 3 Survival curve of SLE patients by hemolytic anemia.

\section{LUPUS NEPHRITIS IN INDIGENOUS AUSTRALIANS: INCIDENCE, HISTOLOGY AND OUTCOME}

1J Nossent*, ${ }^{2} \mathrm{~F}$ Ghazanfari, ${ }^{3} \mathrm{Z}$ Jabbar. ${ }^{1}$ The University of Western Australia, School of Medicine, Perth, Australia; ${ }^{2}$ Maroondah Consulting, Rheumatology, Melbourne, Australia; ${ }^{3}$ Royal Darwn Hospital, Nephrology, Darwin, Australia

\subsection{6/lupus-2017-000215.413}

Background and aims Ethnicity impacts on disease frequency and severity in patients with Lupus Nephritis (LN). As such data are lacking for Indigenous Australians (IA), we investigated the epidemiology, histopathology and outcomes of biopsy proven LN in the Top End of the Northern Territory, Australia.

Methods All patients with biopsy confirmed LN between 2001 and January 2011 were identified from a central database at the Royal Darwin Hospital. Demographics and renal histopathology at first renal biopsy (ISN classification, NIH activity (AI) and chronicity index (CI), immunofluorescence (IF) and electron dense deposit (EDD)) were recorded. Clinical findings and outcomes were retrieved by chart review.

Results The total study cohort $(n=42$, age 30 years; $86 \%$ female) included 31 IA patents (74\%). The annual LN incidence was $7 / 100,000$ in IA patients versus $0.7 / 100,000$ in non-indigenous $(\mathrm{NI})$ patients $(\mathrm{p}<0.01)$.

Proliferative LN (class III+IV) was less frequent in IA patients $(42 \%$ vs. $72 \%) \quad(\mathrm{p}<0.01)$, but AI $(2$ vs. 5, $\mathrm{p}=0.06)$ and CI ( 2 vs. $1, \mathrm{p}=0.9)$ scores did not differ significantly.

Five and ten year patient survival rates were $69 \%$ and $50 \%$ for IA and $90 \%$ for NI patients, while renal survival was $87 \%$ and $53 \%$ in IA patients and $100 \%$ in NI patients. The leading causes of death were infections (38.6\%), end stage renal disease (23\%), cardiovascular events (15.4\%).

Conclusions Biopsy proven LN occurs much more frequently in the IA than in the Causcasion population. While associated with poor patient and renal survival, this is not explained by the histological severity of LN.

\section{PREDICTORS OF END-STAGE RENAL DISEASE IN FILIPINOS WITH LUPUS NEPHRITIS: A CASE-CONTROL STUDY}

B Paras*, E Venegas, S Navarra. University of Santo Tomas Hospital, Internal Medicine, Manila, Philippines

\subsection{6/lupus-2017-000215.414}

Background and aims End-stage renal disease (ESRD) is a major cause of morbidity and mortality in systemic lupus erythematosus (SLE). We studied the risk factors for development of ESRD among Filipino patients with lupus nephritis.

Methods Included were ESRD patients entered in the SLE database of University of Santo Tomas (UST) Hospital, Manila, Philippines from 2005 to 2015. The control group consisted of lupus nephritis patients without ESRD from same database - matched for age, gender and disease duration. Associations of risk factors including comorbidities and treatment for ESRD were tested using Cox-proportional hazard model.

Results Cases included 71 ESRD patients (58, 81.7\% females,

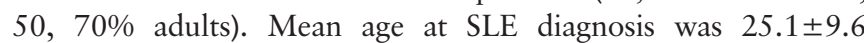
(7-47) years, mean SLE disease duration 9.5 \pm 5.5 (1-29) years, and mean disease duration from SLE diagnosis to ESRD $5.0 \pm 3.0(0.5-14)$ years. By multivariate analysis, hypertension [hazard ratio (HR) 3.754; 95\% CI 2.230, 6.320; p <0.001], and diabetes mellitus (HR 3.775; 95\% CI 1.691, 8.425; $\mathrm{p}<0.001)$ prior to SLE diagnosis were independently associated with progression to ESRD. Onset and treatment of nephritis, and antiphospholipid antibody syndrome (APS) did not significantly impact progression to ESRD. Patients with both hypertension and APS had the shortest disease duration prior to ESRD, but this did not achieve statistical significance.

Conclusions We showed that hypertension and diabetes mellitus prior to SLE diagnosis were strongly associated with progression to ESRD in SLE, reinforcing need to recognise and aggressively control these co-morbidities early at SLE diagnosis, including minimal use of aggravating factors like corticosteroids. 